\title{
On the Energy Efficiency in Multi-user Multi-relay Coded Network
}

\author{
Nan Qi ${ }^{1,2}$, Ming Xiao ${ }^{2}$, Theodoros A. Tsiftsis ${ }^{3,4}$, Phuong L. Cao ${ }^{2}$, Mikael Skoglund ${ }^{2}$, and Lixin $\mathrm{Li}^{1}$ \\ ${ }^{1}$ School of Electronics and Information, Northwestern Polytechnical University, China \\ ${ }^{2}$ School of Electrical Engineering, KTH Royal Institute of Technology, Sweden \\ ${ }^{3}$ Department of Electrical Engineering, Technological Educational Institute of Central Greece, Greece \\ ${ }^{4}$ School of Engineering, Nazarbayev University, Kazakhstan
}

\begin{abstract}
In this paper, the energy efficiency (EE) of a cooperative diversity system with maximum diversity network coding (MDNC) is studied. In the considered system, channel state information (CSI) is only available at the receivers for all the channels. We formulate the problem of maximizing the $\mathrm{EE}$ under the constraint on the outage probability. The problem is NP-hard due to the non-convexity of the outage probability function and the nonlinear fractional structure of the introduced EE. To solve the optimization problem efficiently, first, the outage probability function is tightly approximated as a log-convex form in the high signal-to-noise ratio (SNR) region. Further, based on the fractional programming, we transform the introduced EE into a subtractive-form, which is proved to be a convex form. The tradeoff between outage probability and EE is given. The results show that our power allocation (PA) policy can substantially increase the EE. We show that $\mathrm{EE}$ can be increased if more relays forward the messages. Additionally, we also investigate the effect of the relay locations on the $\mathrm{EE}$ and demonstrate that the increase in the transmission distance in the first hop causes the loss of the EE. The loss can be reduced by our PA policy.
\end{abstract}

\section{INTRODUCTION}

Network coding is proposed to achieve the maximum information flow in multi-user networks, where the user messages are mixed into new codewords at the intermediate relay nodes [1]-[11]. Recently, there are a significant amount of works focusing on the physical layer network coding for the multi-access networks. Based on binary network coding, classical butterfly network and the two-way relay scheme have been widely studied [1]-[5]. Relay selection is conducted to maximize the energy efficiency (EE) [1], minimize the bit error rate [2], [4] and maximize the throughput [3], [5]. In [6], an approach with dynamic network coding has been applied. The authors have analyzed how the number of users and the field size of codes influence the EE for networks with channel state information available at the transmitters (CSIT). To enable more users to cooperate, analog network coding (ANC) is applied. In [7], the packet flow and the channel occupation frames are both scheduled to improve the network throughput. In [8], the EE-maximization problem

The research leading to these results has received funding from the China Scholarship Council (CSC), Fundamental Research Funds for the Central Universities (No. 3102014JCQ01052, 3102014KYJD034, 3103014KYJD040) and the Doctorate Foundation of Northwestern Polytechnical University (CX201317). for ANC-based two-way relay (TWR) networks has been tackled. In [9], the authors have shown that when the number of sources/relays or the modulation order increases, ANC may be more energy efficient than the conventional timeorthogonal non-cooperative transmission scheme (referred to as No Network Coding scenario (NoNC)). However, the above network coding schemes are suboptimal in terms of reliability, especially in the high signal-to-noise ratio (SNR) regions resulting in a suboptimal diversity order. To achieve the full diversity order for a group of cooperative users, the concept of maximum diversity network coding (MDNC) is proposed by Ming Xiao, et al in [10] and [11]. It is shown that, for both orthogonal and non-orthogonal channel cases, an $M$-user $N$-relay network based on MDNC can achieve a diversity of $N-M+1$ if direct source-BS channels are absent, while the dynamic network coding [6] and analog network coding [8] can not [10]. It is also proved in [10] that MDNC can provide the network with a larger outage capacity than the dynamic network coding and analog network coding in the high SNR region.

The works in [10] and [11] are mainly concerned about the outage performance. However, with increasing costs and environmental concerns, the study of energy efficient networks is not only necessary for reducing the energy costs but also for addressing the environmental concerns. EE is a measurement for how efficiently the energy is used in a communication system [12]. To increase the EE, we apply MDNC at the relaying nodes for the multi-user and multirelay (MUMR) network where channel state information (CSI) is only available at the receivers for all the channels. We aim at maximizing the EE meanwhile satisfying the target outage probability. Specifically, PA is optimized among the users and relays according to the channel statistics knowledge and other system parameters. Different from the work in [10], [11], the channel statistics and communication distances for channels are not identical, which yields a completely different mathematical problem. Our optimization problem is considerably more challenging. Even though we show that the optimization problem is NP-hard, we are able to propose a fast and low-complexity algorithm. The effects of both the number of relays and relay locations on the EE are investigated.

The rest of the paper is organized as follows. In Section II, 
we present the system model. Problem formulation is given in Section III. Then, we propose an algorithm in Section IV. The analytical and simulation results are presented in Section V. Section VI concludes this paper.

\section{System MOdeL}

We consider a network deployed with $N$ relays and one base-station (BS). There are $M$ users in the network which transmit their independent messages to the BS via half-duplex relays. We make the following assumptions: (i) Every user has one message to be transmitted and all messages are of the same length ${ }^{1}$, denoted as $|S|$; (ii) All the users and relays transmit information with a fixed rate $\alpha_{0}$ bits per second; ${ }^{2}$ (iii) There is no direct connection between users and the BS due to the long communication distance or physical obstacles.

\section{A. Transmission Scheme}

We assume that all nodes operate in time division multiple access (TDMA). Thus, there is only one transmitter transmitting its message at any time. The similar transmission scheme appear in [6], [13]. The whole transmission consists of two hops as illustrated below.

\section{1) The First Hop: User-relay Transmission}

In the first hop, every user broadcasts its message to relays with a duration of $T_{1}=|S| / \alpha_{0}$ seconds. Let $U_{i}$, $i \in\{1,2, \cdots, M\}$, and $R_{j}, j \in\{1,2, \cdots, N\}$, separately represent the $i$ th user and $j$ th relay. In $U_{i}$ broadcasting phase, $R_{j}$ receives the channel codeword $S_{i}$ from $U_{i}$ as follows:

$$
Y_{i j}=h_{i j} \sqrt{p_{i}} S_{i}+z_{i j},
$$

where $h_{i j} \sim \mathcal{C N}\left(0, d_{i j}^{-n_{i j}} \sigma_{h_{i j}}^{2}\right)$ is the channel gain, which combines path-loss and Rayleigh fading. $d_{i j}^{-n_{i j}}$ and $\sigma_{h_{i j}}^{2}$ denote the path loss and variance of the Rayleigh distribution, respectively; $d_{i j}$ is the distance; $n_{i j}$ is the channel path loss exponent; $p_{i}$ is the transmitting power at $U_{i} ; z_{i j} \sim \mathcal{N}\left(0, N_{0, i j} B\right)$ denotes the additive Gaussian white noise; $N_{0, i j}$ is the onesided power spectral density of white Gaussian noise and $B$ is the bandwidth.

The achievable rate for the channel between $U_{i}$ and $R_{j}$ is

$$
C_{i j}=B \log _{2}\left(1+\frac{\left|h_{i j}\right|^{2} p_{i}}{N_{0, i j} B}\right),
$$

where $\left|h_{i j}\right|$ is the amplitude of $h_{i j}$.

After user transmission, the activated relay, e.g., $R_{j}$, will try to decode $Y_{i j}, \forall i \in\{1,2, \cdots, M\}$, and the result is denoted as $\hat{S}_{i j}$. An outage event occurs in the channel between $U_{i}$ and $R_{j}$ when the fixed data transmission rate is larger than the achievable rate, i.e., $C_{i j}<\alpha_{0}$.

\section{2) The Second Hop: Relay-BS Transmission}

The following notations will be used in our description of the transmission process.

\footnotetext{
${ }^{1}$ We note that this assumption is made for the simple expression. The system model can be directly extended to general cases by assigning different number of messages with different lengths to different users.

${ }^{2}$ The transmission rate on all the links are not necessarily the same. We make the assumption only for simplifying expression.
}

$\Theta:$ the index set of all the relays.

$\Phi_{K}$ : Suppose $K$ relays succeed in receiving and decoding all the user messages in the first hop. The index set is $\Phi_{K}$. Note that $K=0$ means that no relay receives and decodes all the user messages.

$\psi_{\tau}$ : the index set of $\tau, \tau \in\{0,1,2, \cdots, N\}$, relays forwarding messages to the BS in the second hop. $\tau=0$ means no relay forwarding messages to the BS.

Clearly, $\psi_{\tau} \subseteq \Phi_{K} \subseteq \Theta$.

If $R_{j}$ fails to decode any of the user messages, it will not forward messages. ${ }^{3}$ Otherwise, if it can decode all source messages, a network coding scheme based on pre-defined MDNC coding coefficients will be applied. A network codeword $W_{j}$ is generated at $R_{j}$ by the linear combination of $\hat{S}_{1 j}, \hat{S}_{2 j}, \cdots, \hat{S}_{M j} . W_{j}$ is given as

$$
W_{j}=\sum_{i=1}^{M} t_{i j} \hat{S}_{i j}, \forall i \in\{1,2, \cdots, M\}, \forall j \in \Phi_{K},
$$

where $t_{i j}$ is the global encoding kernel for $\hat{S}_{i j}$ at relay $R_{j}$. $t_{i j}$ constitutes the transfer matrix $\mathbf{T}_{M \times N}$ corresponding to MDNC

$$
\mathbf{T}_{M \times N}=\left(\begin{array}{cccc}
t_{11} & t_{12} & \ldots & t_{1 N} \\
t_{21} & t_{22} & \ldots & t_{2 N} \\
& \ldots & \ldots & \\
t_{M 1} & t_{M 2} & \ldots & t_{M N}
\end{array}\right)
$$

$\mathbf{T}_{M \times N}$ has a rank- $M$ for any $M$ columns [10]. ${ }^{4}$ Then $W_{j}$ will be forwarded to the BS. Note that with network coding, we have $\left|W_{j}\right|=\left|\hat{S}_{i j}\right|=|S|, \forall i, j$. Correspondingly, every transmission in the second hop lasts the same duration, i.e., $T_{1}$ seconds. The received message at the BS can be written as

$$
\hat{W}_{j}=g_{j} \sqrt{p_{j}^{\prime}} W_{j}+z_{j}, \forall j \in \psi_{\tau},
$$

where $g_{j} \sim \mathcal{C N}\left(0, d_{j}^{-n_{j}} \sigma_{g_{j}}^{2}\right)$ is the channel gain; $d_{j}^{-n_{j}}$ and $\sigma_{g_{j}}^{2}$ denote the path loss and variance of the Rayleigh distribution, respectively; $d_{j}$ is the distance; $n_{j}$ is the channel path loss exponent. $p_{j}^{\prime}$ is the transmitting power at relay $R_{j}$; $z_{j} \sim \mathcal{N}\left(0, N_{0, j} B\right)$ is the noise term and $N_{0, j}$ is the power spectral density of noise. Note that $W_{j}$ is also protected by channel coding. To simplify the illustration, we ignore the notation of the channel codeword for $W_{j}$.

Finally, the BS decodes all network codewords from channel decoding and obtain $\hat{W}_{1}, \hat{W}_{2}, \cdots, \hat{W}_{\tau}$. After that, the BS obtains the estimated source messages $\bar{S}_{1}, \bar{S}_{2}, \cdots, \bar{S}_{M}$ jointly from $\hat{W}_{1}, \hat{W}_{2}, \cdots, \hat{W}_{\tau}$ by network decoding.

\footnotetext{
${ }^{3}$ We note that if $R_{j}, \forall j \in \Phi_{K}$, decodes a part of the source messages before forwarding them to the BS, the user messages still can be recovered at the BS. For example, suppose that every relay only manages to decode one separate source message and forward it to the BS, then the BS can recover the source message separately. As we will illustrate later, according to the design rule, some useful messages are dropped such that we obtain the upper bound of the outage probability and the corresponding lower bound of the EE. Here, we make the rigid rule for simplifying the system design, which is also applied in [11].

${ }^{4}$ Since the design of MDNC encoding and decoding are not our main points, we skip their design details.
} 


\section{PROBLEM Formulation}

In what follows, we will investigate the total consumed energy, outage probability and EE.

\section{A. Energy Efficiency}

Since the network decoder either recovers all source messages or cannot decode any source message, the outage probability for all user nodes in the MDNC scenario are the same. Denote the outage probability as $\operatorname{Pr}_{\text {out }}$. Thus the expected number of successfully transmitted information bits $L$ can be expressed as

$$
L=M \alpha_{0} T_{1}\left(1-\operatorname{Pr}_{\text {out }}\right) .
$$

EE is evaluated as $L$ divided by the total consumed energy $E_{\text {tot }}$ in the first and second hop, i.e., EE can be expressed as

$$
\eta_{E E}=\frac{M \alpha_{0} T_{1}\left(1-\operatorname{Pr}_{\text {out }}\right)}{E_{\text {tot }}} .
$$

In the following, we separately give the expression for $E_{t o t}$ and $\operatorname{Pr}_{\text {out }}$.

\section{B. Total Consumed Energy}

The total energy consumptions at the users and relays are separately $\sum_{i=1}^{M} p_{i} T_{1}$ and $\sum_{j=1}^{N} p_{j}^{\prime} T_{1}$. We have

$$
E_{t o t}=\left(\sum_{i=1}^{M} p_{i}+\sum_{j=1}^{N} p_{j}^{\prime}\right) T_{1}
$$

\section{Outage Probability}

Since MDNC is used, any $M$ out of $\tau$ network codewords can be used to recover the $M$ user messages at the BS. An outage event happens when fewer than $M$ network codewords are received by the $\mathrm{BS}$, i.e., $\tau \leq M$ [10]. Thus we require that in every transmission, the number of transmitters should be less than the number of relays, i.e., $N$. If the number of users exceeds $N$, we will split the users into several groups and transmission proceeds group by group. For convenience, we assume $M<N$.

An outage event happens in the following two cases in terms of $K$.

\section{Case $\mathcal{A}: K<M$}

In this case, user messages cannot be recovered regardless of how the second hop proceeds. Let $\operatorname{Pr}\left\{\zeta_{K}\right\}$ denote the probability that all the relays in set $\Phi_{K}$ successfully receive all the user messages in the first hop. The outage event happens when $K<M$ with probability $\operatorname{Pr}_{\text {out }, \mathcal{A}}$, i.e.,

$$
\operatorname{Pr}_{\text {out }, \mathcal{A}}=\sum_{K=0}^{M-1} \operatorname{Pr}\left\{\zeta_{K}\right\}
$$

Let $\rho_{j}$ represent the probability that $R_{j}$ can successfully recover all $M$ user messages in the first hop, which can be represented as

$$
\rho_{j}=\prod_{i=1}^{M}\left(1-\operatorname{Pr}_{e, i j}\right)
$$

where $\operatorname{Pr}_{e, i j}$ is the outage probability of $U_{i}-R_{j}$ end-to-end transmission and can be calculated as

$\operatorname{Pr}_{e, i j}=\operatorname{Pr}\left\{C_{i j}<\alpha_{0}\right\}=\operatorname{Pr}\left\{\left|h_{i j}\right|^{2}<\frac{\left(2^{\alpha_{0} / B}-1\right) N_{0, i j} B}{p_{i}}\right\}$.

Since $x_{i j}=\left|h_{i j}\right|^{2}$ follows the exponential probability density function (PDF) [13]

$$
f\left(x_{i j}\right)=\frac{1}{\sigma_{h_{i j}}^{2} d_{i j}^{-n_{i j}}} \exp \left(-\frac{x_{i j}}{\sigma_{h_{i j}}^{2} d_{i j}^{-n_{i j}}}\right),
$$

(7) can be rewritten as

$$
\operatorname{Pr}_{e, i j}=1-\int_{\frac{\left(2^{\alpha_{0} / B}-1\right) N_{0, i j} B}{p_{i}}}^{+\infty} f\left(x_{i j}\right) d x_{i j}=1-\exp \left(-\frac{c_{i j}}{p_{i}}\right),
$$

where $c_{i j}=\frac{\left(2^{\alpha_{0} / B}-1\right) N_{0, i j} B}{d_{i j}^{-n_{i j}} \sigma_{h_{i j}}^{2}}>0$. Then, $\operatorname{Pr}\left\{\zeta_{K}\right\}$ in (5) can be represented as

$$
\operatorname{Pr}\left\{\zeta_{K}\right\}=\sum_{\Phi_{K}}\left(\prod_{j \in \Phi_{K}} \rho_{j} \prod_{j \in \Theta \backslash \Phi_{K}}\left(1-\rho_{j}\right)\right),
$$

where $\sum_{\Phi_{K}}(\beta)$ represents the sum of $\beta$ when $\Phi_{K}$ is in different cases. $\Phi_{K}$ consists of $K$ relays randomly chosen from the activated $N$ relays, including $C_{N}^{K}$ cases.

2. Case $\mathcal{B}: \quad K \geq M$

In this case, an outage event happens when the number of relays forwarding the codewords to the BS in the second hop is smaller than $M$.

Let $\operatorname{Pr}_{\text {out }, \mathcal{B}}$ denote the outage probability in case $\mathcal{B}$, given by

$$
\operatorname{Pr}_{\text {out }, \mathcal{B}}=\sum_{K=M}^{N}\left(\operatorname{Pr}\left\{\zeta_{K}\right\} \cdot \sum_{\tau=0}^{M-1} \operatorname{Pr}\left\{\varsigma_{\tau} \mid \zeta_{K}\right\}\right),
$$

where $\operatorname{Pr}\left\{\zeta_{K}\right\}$ is given in (9); $\operatorname{Pr}\left\{\varsigma_{\tau} \mid \zeta_{K}\right\}$ represents the probability that any combination of $\tau$ relays in $\Phi_{K}$ successfully transmit the user message in the second hop, which is given as

$$
\operatorname{Pr}\left\{\varsigma_{\tau} \mid \zeta_{K}\right\}=\sum_{\psi_{\tau}}\left(\prod_{j \in \psi_{\tau}}\left(1-\operatorname{Pr}_{e, j}\right) \prod_{j \in \Phi_{K} \backslash \psi_{\tau}} \operatorname{Pr}_{e, j}\right),
$$

where $\sum_{\psi_{\tau}}(\varpi)$ represents the sum of $\varpi$ when $\psi_{\tau}$ is in different cases. $\psi_{\tau}$ is the set of $\tau$ indexes randomly chosen from $\Phi_{K}$, including $C_{K}^{\tau}$ cases. $\operatorname{Pr}_{e, j}$ is the outage probability for the transmission between $R_{j}$ and the BS. Similar to the calculation of $\operatorname{Pr}_{e, i j}, \operatorname{Pr}_{e, j}$ can be evaluated as

$$
\operatorname{Pr}_{e, j}=1-\exp \left(-\frac{c_{j}}{p_{j}^{\prime}}\right)
$$

where $c_{j}=\frac{\left(2^{\alpha_{0} / B}-1\right) N_{0, j} B}{d_{j}^{-n_{j}} \sigma_{g_{j}}^{2}}>0$.

Since cases $\mathcal{A}$ and $\mathcal{B}$ are mutually independent, the outage probability can be calculated as

$$
\operatorname{Pr}_{\text {out }}=\operatorname{Pr}_{\text {out }, \mathcal{A}}+\operatorname{Pr}_{\text {out }, \mathcal{B}} \text {. }
$$




\section{Optimization Problem Formulation}

We have the following formulation:

$$
\begin{array}{ll}
\mathbf{P 1}: & {\left[\mathbf{P}_{\mathbf{S}}^{*}, \mathbf{P}_{\text {MDNC }}^{*}\right]=\underset{\left[\mathbf{P}_{\mathbf{s}}, \mathbf{P}_{\mathbf{M D N C}}\right]}{\operatorname{argmax}} \eta_{E E}} \\
\text { s.t. } & \\
& 0<p_{i} \leq P_{S, \max }, \forall i \in\{1,2, \cdots, M\}, \\
& 0 \leq p_{j}^{\prime} \leq P_{R, \max }, \forall j \in\{1,2, \cdots, N\}, \\
& \operatorname{Pr}_{\text {out }} \leq \operatorname{Pr}_{\text {out }, \text { target }},
\end{array}
$$

where $(\cdot)^{*}$ represents the optimum solution. $\mathbf{P}_{\mathbf{s}}=$ $\left[p_{1}, p_{2}, \cdots p_{M}\right]$ denotes the vector of the power allocated to users, while $\mathbf{P}_{\mathrm{MDNC}}=\left[p_{1}^{\prime}, p_{2}^{\prime}, \cdots p_{N}^{\prime}\right]$ denotes the vector of the power allocated to relays.

The product form in (9)-(11) and nonlinear fractional structure of $\eta_{E E}$ make $\mathbf{P 1}$ complicated and challenging. With Brute-force algorithm, the computation complexity grows exponentially with the size of network scale, i.e. $(M+N)$, which is unacceptable when the network is large. To solve $\mathbf{P 1}$ efficiently, we explore a fast algorithm in Section IV.

\section{Approximated-optimal Power Allocation POLICY}

To solve P1 efficiently, we can convert P1 into a convex optimization problem allowing for the adoption of efficient interior-point method. The detailed steps can be described as follows.

\section{Step 1: Geometric Programming Approximation of $\operatorname{Pr}_{\text {out }}$}

To obtain the outage probability expression, we first give the closed-form expressions for $\rho_{j},\left(1-\operatorname{Pr}_{e, j}\right),\left(1-\rho_{j}\right)$ and $\operatorname{Pr}_{e, j}$. By substituting (8) into (6), we have

$$
\rho_{j}=\exp \left(-\sum_{i=1}^{M} c_{i j} / p_{i}\right), 1-\operatorname{Pr}_{e, j}=\exp \left(-c_{j} / p_{j}^{\prime}\right) .
$$

For high SNR regions, i.e., when $c_{i j} / p_{i} \rightarrow 0, c_{j} / p_{j}^{\prime} \rightarrow 0$, we have $\exp \left(-c_{i j} / p_{i}\right) \sim 1$ and $\exp \left(-c_{j} / p_{j}^{\prime}\right) \sim 1$. Furthermore, we have

$$
\rho_{j} \sim 1,\left(1-\operatorname{Pr}_{e, j}\right) \sim 1 .
$$

Moreover, since $\lim _{x \rightarrow 0} 1-\exp (-x)=x$, we have

$$
1-\rho_{j}=1-\exp \left(-\sum_{i=1}^{M} c_{i j} / p_{i}\right) \approx \sum_{i=1}^{M} \frac{c_{i j}}{p_{i}} .
$$

$\operatorname{Pr}_{e, j}=1-\exp \left(-c_{j} / p_{j}^{\prime}\right)$ should be 1 which $p_{j}^{\prime}=0$. However, using the approximation $\lim _{x \rightarrow 0}(1-\exp (-x))=x$ will lead $\operatorname{Pr}_{e, j}$ infinitely large, which is obviously not valid. Instead, we take the approximation $1-e^{-x} \sim \frac{x}{x+1}$ and have

$$
\operatorname{Pr}_{e, j}=\frac{c_{j}}{c_{j}+p^{\prime}}{ }_{j}
$$

Note that $\operatorname{Pr}_{e, j}$ in (21) is 1 when $p_{j}^{\prime}=0$, which is reasonable. By substituting (19) - (21) into (9) - (13), we rewrite $\operatorname{Pr}_{\text {out }}$ as (22), which is a tight approximation for high SNR. Note that
(22) is in the geometric programming form of $p_{i}$ and $\left(p_{j}^{\prime}+c_{j}\right)$. Further, we introduce two new variables, i.e., $\tilde{p}_{i}$ and $\tilde{p}_{j}^{\prime}$ here. We have

$$
p_{i}=e^{\tilde{p}_{i}}, \quad p_{j}^{\prime}+c_{j}=c_{j} e^{\tilde{p}_{j}^{\prime}} .
$$

Then, (15), (16) and (22) can be rewritten as (25), (26) and (23), respectively.

$$
\begin{aligned}
& 0<p_{i}=e^{\tilde{p}_{i}} \leq P_{S, \max }, \\
& 0 \leq p_{j}^{\prime}=c_{j} e^{\tilde{p}_{j}^{\prime}}-c_{j} \leq P_{R, \max } .
\end{aligned}
$$

Step 2: Fractional Programming for the Objective Function

We have the following theorem for solving P1 [15].

Lemma 1. The resource allocation policy can achieve the maximum energy efficiency $q^{*}=\max \eta_{E E}$, if and only if

$$
V\left(q^{*}\right)=\max \left\{M \alpha_{0} T_{1}\left(1-\operatorname{Pr}_{\text {out }}\right)-q^{*} E_{\text {tot }}\right\}=0,
$$

$V\left(q^{*}\right)$ is referred to as the subtractive-form of the primal objective function. According to Lemma 1, P1 can be reformulated as finding the optimum transmitted powers at users and relays to satisfy

$$
\max \left\{V=\left(M \alpha_{0} T_{1}\left(1-\operatorname{Pr}_{\text {out }}\right)-q E_{\text {tot }}\right)\right\}=0,
$$

In the Dinkelbach's method, $q$ is iteratively updated. In every iteration, it addresses (28) with a given $q$ and then judges whether $V(q)$ converges to a given tolerance. If not, $q$ is updated and repeat the maximization problem until it converges or reaches the maximal iterations. The details can be found in [15]. Note that with given $q$ in every iteration, we have

$$
\max V \Leftrightarrow \min -V \Leftrightarrow \min V^{\prime} \stackrel{(a)}{\Leftrightarrow} \min \tilde{V},
$$

where " $\Leftrightarrow$ means the expression in the left side is equivalent to that in the right side. $V^{\prime}=-V+M \alpha_{0} T_{1}+q T_{1} \sum_{j=1}^{N} c_{j}$ and $\tilde{V}=\log \left(V^{\prime}\right)$. (a) satisfies with the fact that $V^{\prime}>0$. Then (28) is equivalent to

$$
\min \{\tilde{V}\}=\log \left(M \alpha_{0} T_{1}+q T_{1} \sum_{j=1}^{N} c_{j}\right) .
$$

By taking $\log$ for both sides of (17), we have P2 shown below. $q^{*}$ and $\left[\tilde{\mathbf{P}}_{\mathbf{s}}^{*}, \tilde{\mathbf{P}}_{\text {MDNC }}^{*}\right]$ are the solutions of $\mathbf{P 2}$ that also satisfy (30).

$$
\begin{aligned}
& \text { P2 }: \min \tilde{V} \\
& \text { s.t. }(25),(26), \\
& \quad \log \left(\operatorname{Pr}_{\text {out }}\right) \leq \log \left(\operatorname{Pr}_{\text {out }, \text { target }}\right) .
\end{aligned}
$$

For P2, we have the following theorem.

Theorem 1. Given $q$ in every iteration, P2 is a convex optimization problem with respect to $\tilde{p}_{i}, \forall i \in\{1,2, \cdots, M\}$, and $\tilde{p}_{j}^{\prime}, \forall j \in \Theta$.

Proof. See the Appendix.

In Algorithm 1, we summarize the algorithm. 


$$
\begin{aligned}
& \operatorname{Pr}_{\text {out }} \\
& \approx \sum_{K=0}^{M-1} \sum_{\Phi_{K}}\left(\prod_{j \in \Theta \backslash \Phi_{K}} \sum_{i=1}^{M} \frac{c_{i j}}{p_{i}}\right)+\sum_{K=M}^{N}\left(\left(\sum_{\Phi_{K}}\left(\prod_{j \in \Theta \backslash \Phi_{K}}\left(\sum_{i=1}^{M} \frac{c_{i j}}{p_{i}}\right)\right)\right) \cdot\left(\sum_{\tau=0}^{M-1} \sum_{\Phi_{K}}\left(\prod_{j \in \Phi_{K} \backslash \psi_{\tau}} \frac{c_{j}}{p_{j}^{\prime}+c_{j}}\right)\right)\right) \\
& =\sum_{K=0}^{M-1} \sum_{\Phi_{K}}\left(\prod_{j \in \Theta \backslash \Phi_{K}} \sum_{i=1}^{M} c_{i j} e^{-\tilde{p}_{i}}\right)+\sum_{K=M}^{N}\left(\left(\sum_{\Phi_{K}}\left(\prod_{j \in \Theta \backslash \Phi_{K}}\left(\sum_{i=1}^{M} c_{i j} e^{-\tilde{p}_{i}}\right)\right)\right) \cdot\left(\sum_{\tau=0}^{M-1} \sum_{\Phi_{K}}\left(\prod_{j \in \Phi_{K} \backslash \psi_{\tau}} e^{-\tilde{p}_{j}^{\prime}}\right)\right)\right) .
\end{aligned}
$$

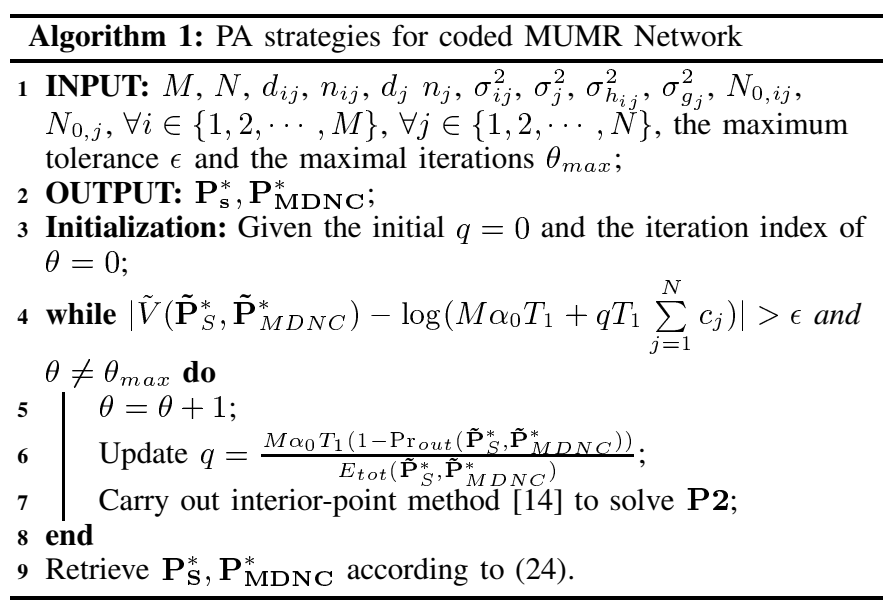

\section{Numerical Results}

In what follows, we give numerical results. The following parameters are assumed. $\sigma_{\mathbf{h}}$ and $\sigma_{\mathbf{g}}$ denote the variance matrics of the Rayleigh fading gains. $\mathbf{N}_{\mathbf{0}, \mathbf{h}}$ and $\mathbf{N}_{\mathbf{0}, \mathbf{g}}$ represent the power spectrum density matrices, which are measured in Watts/Hz. $d_{h}$ and $d_{g}$ represent the distance matrices which are measured in meter. $\mathbf{n}_{\mathbf{h}}$ and $\mathbf{n}_{\mathbf{g}}$ represent the path-loss exponent matrices. Specifically, elements at the $i$ th row and the $j$ th column of $\sigma_{\mathbf{h}}, \mathbf{d}_{\mathbf{h}}, \mathbf{n}_{\mathbf{h}}$ and $\mathbf{N}_{\mathbf{0}, \mathbf{h}}$ correspond to the parameters for the $U_{i}-R_{j}$ channel. Elements at the $j$ th columns in $\sigma_{\mathbf{g}}, \mathbf{d}_{\mathbf{g}}, \mathbf{n}_{\mathbf{g}}$ and $\mathbf{N}_{\mathbf{0}, \mathbf{g}}$ correspond to the parameters for the $R_{j}$-BS channel. The randomly generated values for all the above parameters are as follows.

$$
\begin{aligned}
& \sigma_{\mathrm{h}}=\left[\begin{array}{llll}
5.1291 & 3.5040 & 4.3367 & 1.1597 \\
4.6048 & 0.9505 & 7.0924 & 0.7808
\end{array}\right] \\
& \sigma_{\mathrm{g}}=\left[\begin{array}{llll}
5.0213 & 4.6821 & 3.4823 & 0.8667
\end{array}\right], \\
& \mathbf{d}_{\mathbf{h}}=\left[\begin{array}{cccc}
857.5 & 457.6 & 927.1 & 840.2 \\
1064.8 & 990.5 & 435.3 & 161.8
\end{array}\right] \text {, } \\
& \mathbf{n}_{\mathbf{h}}=\left[\begin{array}{llll}
2.5570 & 2.9150 & 2.3152 & 3.0143 \\
3.0938 & 3.1298 & 2.6412 & 2.9708
\end{array}\right] \text {, } \\
& \mathbf{d}_{\mathrm{g}}=\left[\begin{array}{llll}
321.7 & 895.7 & 752.2 & 929.4
\end{array}\right] \text {, } \\
& \mathbf{n}_{\mathbf{g}}=\left[\begin{array}{llll}
2.8006 & 2.2838 & 2.8435 & 2.5315
\end{array}\right], \\
& \mathbf{N}_{\mathbf{0}, \mathbf{h}}=10^{-14}\left[\begin{array}{cccc}
0.063 & 0.035 & 0.27 & 0.003 \\
0.001 & 0.001 & 0.214 & 0.548
\end{array}\right] \text {, } \\
& \mathbf{N}_{\mathbf{0}, \mathbf{g}}=10^{-14}\left[\begin{array}{llll}
0.1900 & 0.3621 & 0.0132 & 0.0612
\end{array}\right] \text {. }
\end{aligned}
$$

We assume that $M=2, N=4, P_{S, \max }=10$ Watts, $P_{R, \max }=20$ Watts, $B=125 \mathrm{KHz}, \alpha_{0}=300 \mathrm{~K}$ and

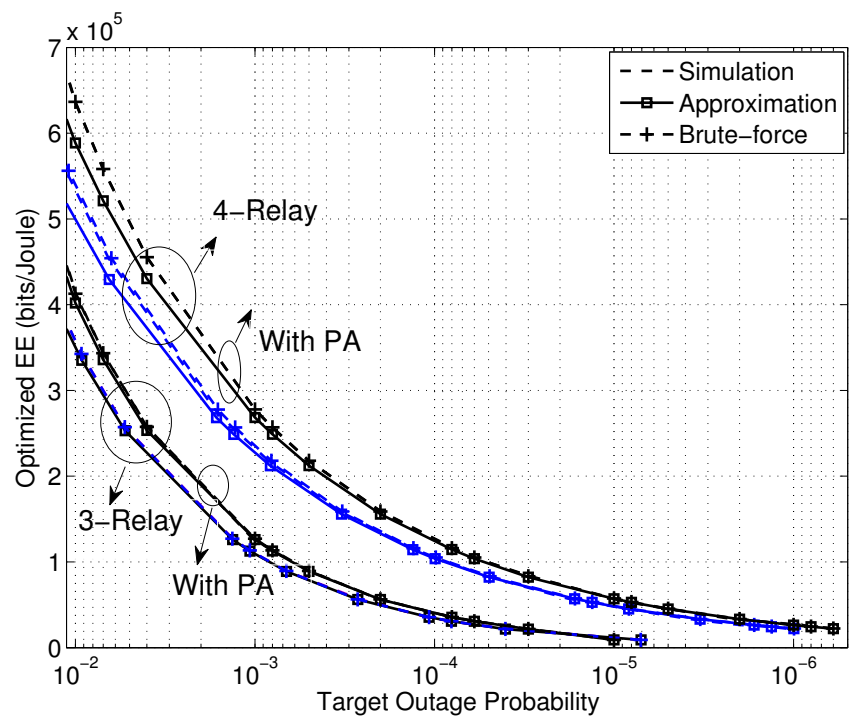

$|S|=125 K$.

Fig. 1. Tradeoff between outage probability and EE.

\section{A. Effects of PA Policy and the Number of Relays}

For comparison, in Fig. 1, we plot the tradeoff curves between $\operatorname{Pr}_{\text {out }}$ and $\eta_{E E}$ for the 4-relay and 3-relay scenarios. The numerical results are obtained by Algorithm 1, the Brute-force algorithm and simulations, respectively. The simulation results are obtained by separately averaging the outage probability, total consumed energy and EE over $10^{8}$ random realizations of the Rayleigh fading channels. It can be observed that the analytical results obtained from Algorithm 1 closely match the results from the Brute-force algorithm and simulation, especially in the low $\operatorname{Pr}_{\text {out }}$ region where higher SNR is needed. All these show that the analytical results obtained by Algorithm 1 are valid.

In Fig. 1, the gaps between the curves for the PA and uniform power policies show the improved EE by PA. It is clear that for the same $\operatorname{Pr}_{\text {out }}$, more than $17 \%$ energy efficiency gains are obtained for both the 3 and 4-Relay scenarios. We can also observe that the EE with 4 Relays is higher than that of 3-Relay scenario. The numerical results also imply that with more relays forwarding messages, the achievable outage probability region is enlarged. This is due to the higher diversity order provided by more relays.

\section{B. Effects of the Relay Locations}

To investigate the effect of the relay locations on the EE, we increase the transmission distance in the first hop and 


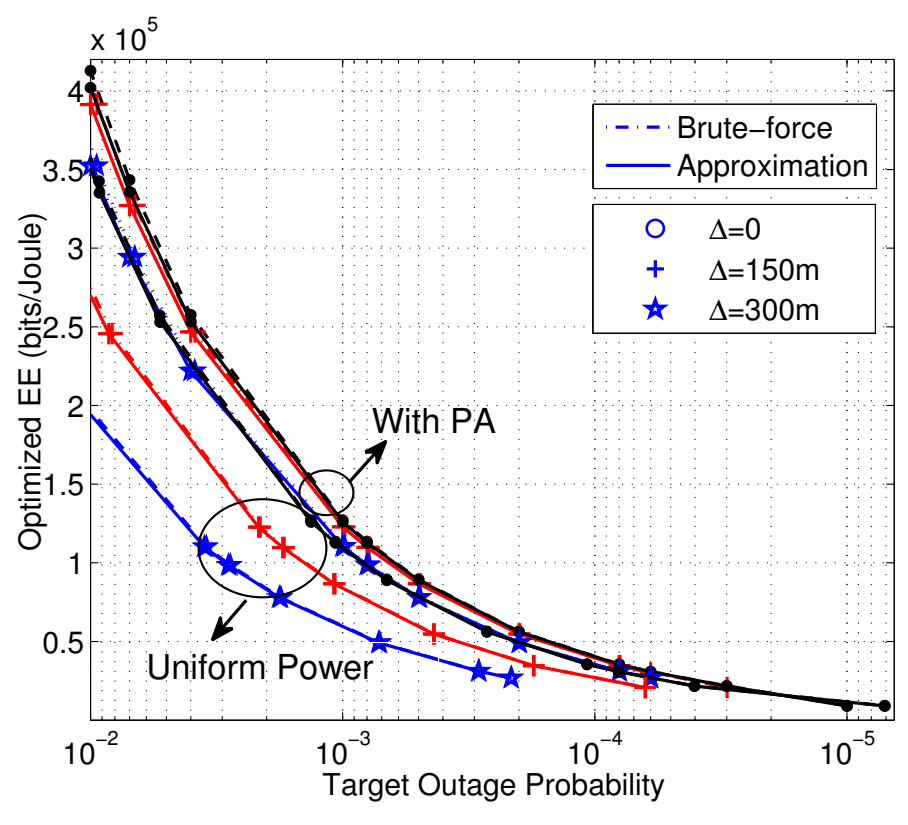

Fig. 2. 3-Relay scenario; Tradeoff between outage probability and EE.

decrease the distance in the second hop. That is, we move the relays farther away from the users and closer to the BS. To be specific, the distance between $U_{i}, \forall i$, and $R_{j}, \forall j$, is changed into $\left(d_{i j}+\Delta\right)$ meters while the distance between $R_{j}$ and the BS is reduced into $\left(d_{j}-\Delta\right)$ meters, where $\Delta$ can be regarded as the shifting distance of one relay. In Fig. 2 , we draw the tradeoff curves for PA and uniform power policies with various shifting distances in 3-Relay scenario. It can be found that an increasing $\Delta$ yields the decrease in the $\mathrm{EE}$, especially for the uniform power cases. It is because the first hop transmission is dominant in the two-hop transmission scheme. The increase in the transmission distance of the first hop deteriorates the outage performance, which needs more energies in the second hop to compensate.

Additionally, it can be noticed that with PA, higher EE gains are achieved for the larger $\Delta$ cases. Specifically, $17 \%, 40 \%$ and $85 \%$ of EE gains are separately obtained for $\Delta=0$, 150 and 300 meters scenarios. From another perspective, the gaps among the tradeoff curves for the three different shifting distances cases with PA are considerably smaller than those with the uniform power cases. It demonstrates that the EE loss resulting from the larger $\Delta$ can be significantly reduced with our proposed PA policy.

\section{CONClusions}

We have studied the energy efficiency for MDNC-based MUMR networks without CSIT. We convert the PA problem into a convex one by applying geometric programming for the outage probability and fractional programming for the EE. The optimal EE-outage probability tradeoff for PA is obtained and analyzed. Analytical results show that PA can sustainably improve the EE. We conclude that the EE can be increased with more relays forwarding the messages. Moreover, it is demonstrated that increasing transmission distance in the first hop results in a decrease in the EE, which can be compensated to a large extent by our PA policy. Our geometric programming approximation for the outage probability can be applied in various communication scenarios, like TWR, ANC, orthogonal frequency-division multiplexing (OFDM) and other systems.

\section{APPENDIX}

Proof. It is obvious that (25) and (26) are convex.

In every iteration of $q$,

$$
\begin{aligned}
V^{\prime} & =M \alpha_{0} T_{1} \operatorname{Pr}_{\text {out }}+q E_{\text {tot }}+q T_{1} \sum_{j=1}^{N} c_{j} \\
& =M \alpha_{0} T_{1} \operatorname{Pr}_{\text {out }}+q T_{1}\left(\sum_{i=1}^{M} e^{\tilde{p}_{i}}+\sum_{j=1}^{N} c_{j} e^{\tilde{p}_{j}^{\prime}}\right),
\end{aligned}
$$

which is the sum of multiple exponential terms multiplied by positive constants. Then their $\log$-form i.e., $\tilde{V}$ is convex [14]. The proof of the convexity property of (31) follows the same approach. Thus, $\mathbf{P 2}$ is a convex optimization problem.

\section{REFERENCES}

[1] K. Xiao, Y. Zhang, G. Feng, X. M. Duan, J. Zhu and Z. Y. Liu, "eCOPE: Energy Efficient Network Coding Scheme in Multi-rate Wireless Networks," Wireless Communications and Networking Conference Workshops (WCNCW), IEEE, pp. 18-23, Apr. 2013.

[2] T. Oechtering and $H$. Boche, "Bidirectional regenerative half-duplex relaying using relay selection," IEEE Trans. Wireless Commun., vol. 7 , no. 5, pp. 1879-1888, 2008.

[3] M. Xiao and T. Aulin, "Optimal decoding and performance analysis of a noisy channel network with network coding," IEEE Trans. Commun., vol. 57, pp. 1402-1412, May 2009.

[4] W. Chen, K. Letaief, and Z. Cao, "Opportunistic network coding for wireless networks," in IEEE International Conference on Communications (ICC), pp. 4634-4639, Jun. 2007.

[5] L. Ding, M. Tao, F. Yang, and W. Zhang, "Joint scheduling and relay selection in one-and two-way relay networks with buffering," in Proc. of IEEE ICC09, Jun. 2009.

[6] G. Y. Zhang and Y. H. Li, "Cooperative multicast transmission strategy for energy-efficient dynamic network coding," Communications Workshops (ICC), IEEE International Conference on, pp. 479-483, Jun. 2013.

[7] W. Mao, X. Wang, A. Tang, H. Qian, "ANC-ERA: Random Access for Analog Network Coding in Wireless Networks," in Mobile Computing, IEEE Transactions on, vol. 15, no. 1, pp. 45-59, Jan. 2016.

[8] M. Zhao, Z. Zhang, W. Y. Zhou and J. K. Zhu, "Maximizing energy efficiency in analog network coding based two-way relay-assisted system," Wireless Communications and Signal Processing (WCSP), Sixth International Conference on, pp. 1-6, Oct. 2014.

[9] K. Ntontin, M. D. Renzo, A. Perez-Neira, C. Verikoukis, “ Is Analog Network Coding more energy efficient than TDMA?" in Computer Aided Modeling and Design of Communication Links and Networks (CAMAD), 2014 IEEE 19th International Workshop on, pp. 244-248, Dec. 2014.

[10] M. Xiao, J. Kliewer and M. Skoglund, "Design of Network Codes for Multiple-User Multiple-Relay Wireless Networks," IEEE Trans. Communn., vol. 60, no. 12, pp. 3755-3766, Dec. 2012.

[11] M. Xiao and M. Skoglund, "Multiple-user cooperative communications based on linear network coding," IEEE Trans. Communn., vol. 58, pp. 3345-3351, Dec. 2010.

[12] S. Verdú. "On Channel Capacity per Unit Cost," IEEE Trans. Inf. Theory, vol. 36, no. 5, pp. 1019-1930, Jan. 1986.

[13] M. Chen, S. Serbetli and A. Yener, "Distributed Power Allocation Strategies for Parallel Relay Networks," IEEE Trans. Wireless Commun., vol. 7, pp. 552-561, Oct. 2011.

[14] S. Boyd and L. Vandenberghe, Convex Optimization, Cambridge University Press, 2004.

[15] W. Dinkelbach, "On nonlinear fractional programming," Manage. Sci., vol. 13, no. 7, pp. 492-498, Mar. 1967. 